\title{
RENDIMIENTO DE DOS VARIEDADES MEJORADAS DE FRIJOL, SEMBRADOS AL VOLEO Y AL ESPEQUE, EN MOSS PAM, WASPAM, RIO COCO
}

\author{
Sankara K. Narvaez Ismael ${ }^{[1]}$ \\ Hector R. Marchena Williams ${ }^{[2]}$ \\ William J Watler Reyes, MSc ${ }^{[3]}$ \\ Enrique Cordón S, MA ${ }^{[4]}$
}

\section{Resumen}

Este artículo presenta el comportamiento agronómico del frijol (Phaseolus vulgaris L.) en dos variedades:(DOR-364 y H-46), establecidos a través del análisis de dos métodos de siembra (al voleo y al espeque), para medir la variable rendimiento. El experimento se realizó en la finca académica SNAKY/URACCAN, ubicada en la comunidad Moss Pam, en el municipio de Waspam río Coco, Región Autónoma Atlántico Norte (RAAN). El cultivo de frijol es de gran relevancia en la vida de las comunidades indígenas, ya que constituye al igual que el arroz, el grano de mayor importancia en la dieta familiar. Es de mencionar que las comunidades indígenas de la RAAN utilizan básicamente dos métodos de siembra (al voleo y al espeque); no obstante, siempre los rendimientos son bajos, por lo que se hace necesario el estudio con las condiciones descritas.

Se utilizó un diseño bifactorial en bloques completos al azar, en donde el factor A: equivale a las dos variedades de frijol (DOR-364 y H-46), y el factor B: dos métodos de siembra (al voleo y al espeque). La evaluación se hizo sobre las características morfométricas presentadas en la fase vegetativa y reproductiva del cultivo. El procesamiento de la información se hizo con el programa estadístico InfoStat, indagando desde el análisis del ANAVA y la prueba Kruskal-Wallis. Los resultados mostraron una alta viabilidad germinativa (95\%), con diferencias estadísticas entre tratamientos en las variables altura y diámetro en la etapa vegetativa. En la etapa reproductiva las variables: número de vainas/plantas y granos/vainas/plantas, fueron las que presentaron diferencias estadísticas entre tratamientos, contrario a las variables número de flores/plantas y longitud de vainas/plantas que no mostraron diferencias. Finalmente el ANAVA con un 95 por ciento de confiabilidad encontró diferencias de medias en la variable rendimiento.

\footnotetext{
[1] Ingeniero Agroforestal, Técnico IREMADES. kn8narvaez@gmail.com

[2] Ingeniero Agroforestal. Técnico NITLAPAN-UCA. titrobert87@yahoo.com

[3] Tutor de la investigación y Docente de la Universidad URACCAN. wwatlerr@yahoo.com

${ }^{[4]}$ Asesor de investigaciones en la Carrera de Ing. Agroforestal URACCAN. encordon@yahoo.com
} 
Se recomienda el establecimiento de la variedad H-46 sea sembrada al voleo y la variedad DOR-364 sembrada al espeque, por presentar los mejores rendimientos con 77.63 y $71.33 \mathrm{qq} / \mathrm{mz}$.

Palabras clave: rendimiento del frijol, siembra al voleo y espeque, trópico húmedo, Nicaragua.

\section{Summary}

This article presents the agronomic behavior of the beans (Phaseolus vulgaris $L$.) in two varieties: (DOR-364 and M-46), that were established through the analysis of two planting methods (broadcast and handspike) to measure the production. The experiment was conducted in the academic farm of Snaky/URACCAN, located in the community of Moss Pam, municipality of Waspam, Rio Coco, North Autonomous Atlantic Region (RAAN). The bean crop is of great importance in the lives of indigenous communities, since just as the rice, it constitutes the most important grain in the family's diets. It should be noted that the indigenous communities of the RAAN basically used two methods of planting (broadcast and handspike), however, productions is always low, so is necessary to make the study with the conditions that have been described.

A bifactorial design in randomized complete blocks was used, where the factor $A$ : is equivalent to the two varieties of beans (DOR-364 and H-46), and factor B, is equivalent to two methods of planting (broadcast and handspike). The evaluation was made on the morphometric characteristics presented in the vegetative and reproductive phase of the crop. The information processing was done with the statistical program InfoStat, making an investigation from the analysis of ANAVA and Kruskal-Wallis test.

The results showed high germination viability (95\%), with statistical differences between treatments in the variables: height and diameter, in the vegetative stage. In the reproductive stage the variables: number of pods/plants and seeds/pods/plants, were the one that presented statistical differences between treatments, contrary to the variables: number of flowers/plants and length of pods/plants, that showed no differences. Finally, the ANAVA with 95 percent of reliability found differences in the variable: performance.

Is recommended to plant the variety $\mathrm{H}-46$ using the broadcast method and that DOR-364 variety be planted by handspike, because it present the best production with 77.63 and $71.33 \mathrm{qq} / \mathrm{mz}$.

Keywords: Bean production, broadcast and handspike planting, humid tropics, Nicaragua. 


\section{Introducción}

Las comunidades de la RAAN, basan su subsistencia en la producción de granos básicos, tubérculos, musáceas, ganado mayor y menor, aprovechamiento forestales, y otras actividades económicas destinadas a sus necesidades de primer orden. La producción de frijol es una de las principales actividades y rubro en la economía comunitaria, ya que genera ingresos y empleo, así como es fundamental en la dieta alimenticia.

Desde el punto de vista ambiental y técnico, el cultivo presenta una serie de problemáticas que afectan la producción de frijol en Nicaragua y particularmente la zona húmeda de la RAAN. De acuerdo al INTA ${ }^{[5]}$ (2004), como son:

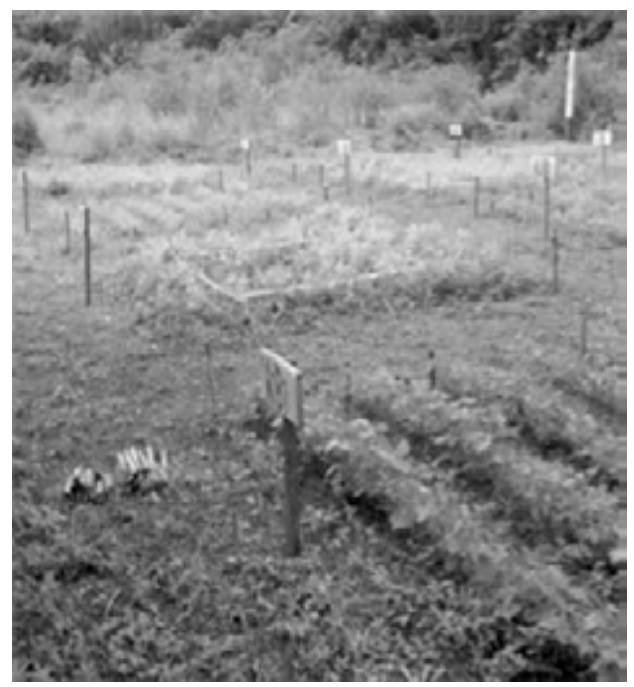

Foto 1. Área de ensayo

- Irregularidad de las lluvias (actualmente el problema es más frecuente).

- Uso de semillas de mala calidad.

- Alta incidencia de enfermedades (ataques de plagas).

- Suelos poco fértiles, y se suma la falta de fertilización.

- Baja densidad de siembra y métodos de siembra inadecuados.

- Mal uso de agroquímicos.

- Ingenuidad en la época de siembra.

- Falta de asistencia técnica y capacitación en el manejo agronómico del cultivo.

Situación que provoca altos costos en la producción, pérdidas parciales en las cosechas y rendimientos muy bajos. Razones que motivaron a desarrollar el presente estudio, con el objetivo de evaluar las dos variedades de frijol más utilizadas por las comunidades indígenas y bajo los dos métodos de siembra tradicional; asimismo, poder caracterizar morfométricamente las etapas del cultivo (vegetativa y reproductiva), subrayar sus tendencias durante su crecimiento y/o desarrollo, las vulnerabilidad y amenazas en cada etapa del ciclo del cultivo.

$\overline{{ }^{[5]} \text { Instituto Nacional Tecnológico Agropecuario }}$ 


\section{Revisión de literatura}

\subsection{Cultivo de frijol (Phaseolus vulgaris L.)}

Desde el punto de vista taxonómico, el frijol es el prototipo del género Phaseolus y su nombre científico es Phaseolus vulgaris L. asignado por Lineo en 1753. Pertenece a la tribu Phaseolae de la subfamilia papilionoidae dentro del orden Rosales y la familia Leguminoseae (CIAT, 1984).

El género Phaseolus incluye aproximadamente 35 especies, de las cuales cuatro se cultivan: P. vulgaris L.; P. lunatus L.; P. coccineus L.; y P. acutifolius A. Gray van latifolius Freeman (CIAT, 1984).

Cuadro No. 1. Taxonomía del cultivo de frijol (Phaseolus vulgaris L.)

\begin{tabular}{|c|c|c|}
\hline No. & Orden & Género \\
\hline 1 & Familia & Rosales \\
\hline 2 & Subfamilia & Leguminoseae \\
\hline 3 & Tribu & Papilionoideae \\
\hline 4 & Subtríbu & Phaseolinae \\
\hline 5 & Género & Phaseolus \\
\hline
\end{tabular}

Fuente: (Barquero et al. 1988).

\subsection{Características de las variables en estudio DOR-364 y H-46}

Los Cuadros No. 2 y No. 3 detallan las características agronómicas de ambas variedades; según el INTA (1996).

Cuadro No. 2. Características agronómicas de la variedad DOR-364

\begin{tabular}{|c|c|}
\hline \multicolumn{2}{|c|}{ Características agronómicas del D0R-364 } \\
\hline Hábito de crecimiento & 1 lb arbustivo guía corta \\
\hline Color de la vaina & Rosada \\
\hline Forma del grano & Alargada \\
\hline Altura de ramificación & $6 \mathrm{~cm}$ \\
\hline Nudos de ramificación & 3.4 \\
\hline Números de ramas & 2.4 \\
\hline Altura del follaje & $50 \mathrm{~cm}$ \\
\hline Ancho del follaje & 54.5 \\
\hline Altura de inserción prime- \\
ra vaina
\end{tabular}




\begin{tabular}{|c|c|}
\hline \multicolumn{2}{|c|}{ Características agronómicas del D0R-364 } \\
\hline Primera flor abierta & 32 días \\
\hline Floración completa & $35-37$ días \\
\hline Llenado de grano & 27 días \\
\hline Madurez fisiológica & $63-68$ días \\
\hline Siembra a cosecha & $75-85$ días \\
\hline Vainas/planta & 12 \\
\hline Granos/vainas & 6 \\
\hline Peso de 10o semillas & $22 \mathrm{gr}$ \\
\hline Rendimiento esperado & $15-25 \mathrm{qq} / \mathrm{Mz}$ \\
\hline Color del grano & Rojo oscuro \\
\hline
\end{tabular}

Cuadro No. 3. Características agronómicas de la variedad H-46

\begin{tabular}{|l|l|}
\hline \multicolumn{2}{|c|}{ Características agronómicas del H-46 } \\
\hline Origen del grano & Honduras \\
\hline Color & Rojo oscuro brillante \\
\hline Color de la vaina & Blanca cuando está madura \\
\hline Forma del grano & Alargada arriñonado \\
\hline Hábito de crecimiento & Arbustiva guía corta, no postrada \\
\hline Número de ramas & 5 \\
\hline Días de floración & $32-36$ días \\
\hline Días de maduración fisiológica & $72-76$ días \\
\hline Días a cosecha & $80-85$ \\
\hline Vainas/planta & $12-18$ \\
\hline Semillas/vainas & $6-7$ \\
\hline pH & $5.6-6.8$ \\
\hline Suelo & Franco, franco arenoso \\
\hline Fecha de siembra & Del 15 de nov., al 15 de dic. \\
\hline
\end{tabular}

\section{Materiales y métodos}

\subsection{Descripción del área de estudio}

El experimento se realizó en la finca académica "Snaki" (108.13 ha) de la universidad URACCAN, situada en la parte alta de la cuenca del río Wawa, en la comunidad Moss Pam, municipio de Waspam, RAAN, Nicaragua. Geográficamente se sitúa entre las coordenadas $14^{\circ} 20^{\prime} 59.9^{\prime \prime}$ Latitud Norte y 8352'18.3" Longitud Oeste (Mapa No.1), con una elevación media de $34 \mathrm{msnm}$, y un relieve que varía de plano a ligeramente ondulado (2-15\% de pendiente) (URACCAN, 2009). 
Mapa 1. Macro-localización de la finca académica "Snaki"

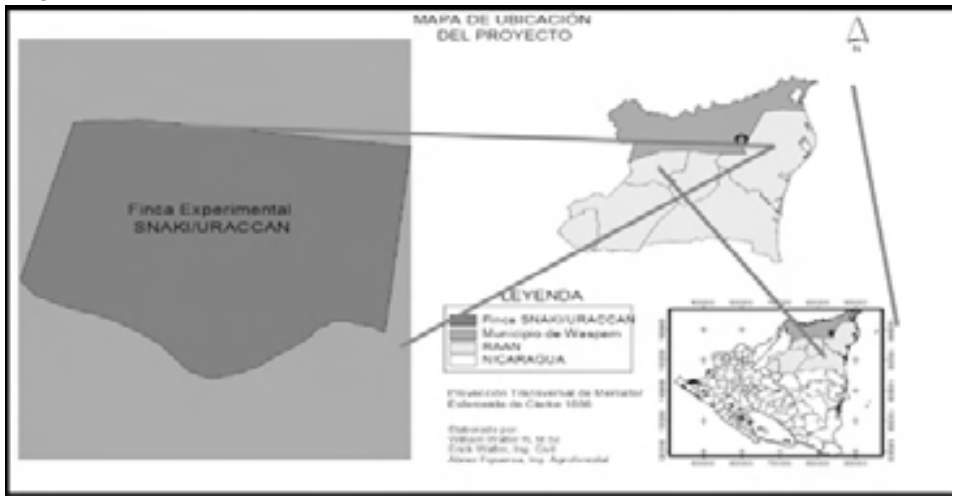

Fuente: Watler (2009)

\subsection{Metodología del estudio}

Etapa 1. Pre-campo: Es la revisión, clasificación y análisis de la información secundaria, que dio origen a la elaboración, presentación y defensa del documento de anteproyecto o guía de investigación.

Etapa 2. Campo: Consistió en la elaboración del cronograma de trabajo de campo por cada objetivo establecido, ajustado a los meses de Enero, Febrero y Marzo del 2010, igualmente se procedió a la preparación del presupuesto y cronograma de actividades de campo y post-campo, incluyendo las actividades de selección, ubicación, medición y preparación de la parcela experimental, como la recolección y revisión de la información de campo.

Etapa 3. Post-campo o procesamiento de la información: Consistió en el ordenamiento de la información de campo, seguido del procesamiento, análisis y discusión de los resultados por objetivos establecidos, hasta finalmente construir las medidas o recomendaciones sobre la base de los resultados obtenidos; esto, con el propósito de contrarrestar los efectos negativos o bajos rendimientos del cultivo de frijol en las comunidades indígenas y mestizas de la región.

\subsection{Universo y muestra}

El universo lo constituyó el área experimental, la cual correspondió a una superficie de $428.75 \mathrm{~m}^{2}$ (17.5 x $\left.24.5 \mathrm{~m}\right)$, con una defensa externa de $2 \mathrm{~m}$ e interna de $1.5 \mathrm{~m}$, una superficie de sub-parcela (P.E) de $14 \mathrm{~m}^{2}(4 \times 3.5 \mathrm{~m})$ y parcela útil (P.U) de $4 \mathrm{~m}^{2}(2 \times 2 \mathrm{~m})$.

Para la siembra al espeque, dentro de cada parcela se trazaron 6 surcos con 22 golpes por metro lineal, en cada golpe se depositaron 3 semillas; proporcionando una densidad por parcela de 396 plantas, para una población inicial de 2,366 plantas. La distancia de siembra correspondió a $60 \mathrm{~cm}$ entre surcos y $15 \mathrm{~cm}$ entre planta. Para el 
caso de la siembra al voleo se tomó 5 manojos de semilla (1,410 semillas aproximadamente), por parcela, la cual suministró un total de 8,460 semillas, regadas en cada parcela, según el diseño experimental.

Figura No. 1. Etapa de desarrollo del cultivo de frijol. Fuente: INTA (2004)

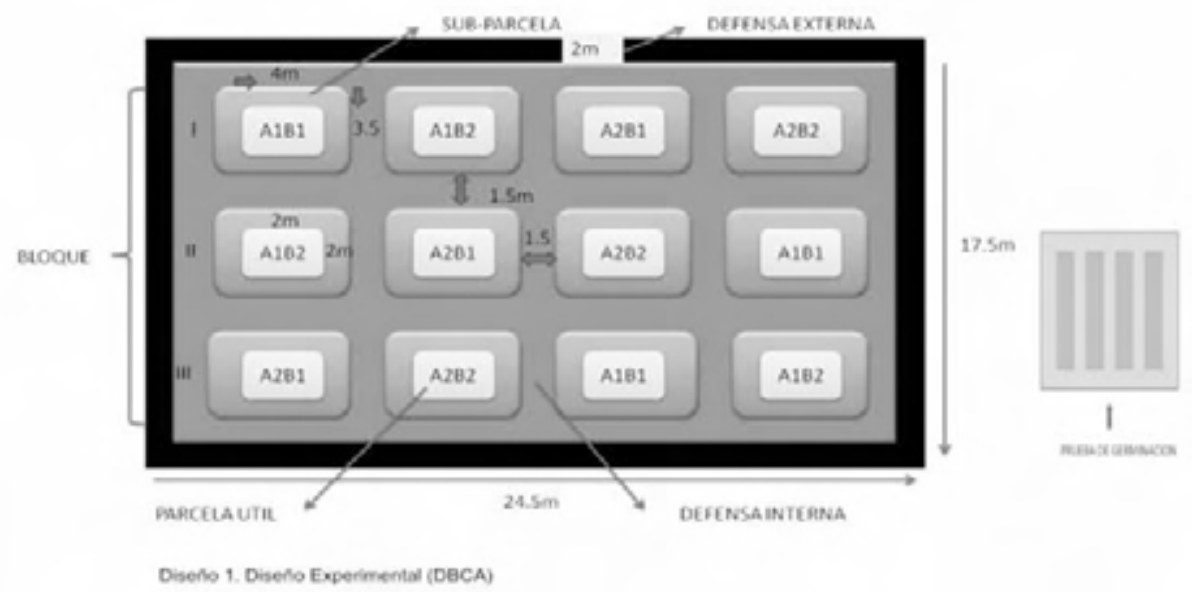

\subsection{Unidades muéstrales}

- Dentro del área experimental se tomaron 6 muestras por cada método, conformando un total de 12 muestras en 3 bloques respectivamente (10 plantas al azar).

- Las evaluaciones se realizaron semanalmente (intervalo de 7 días inmediatamente de la siembra $\left(\mathrm{DDS}^{6}\right)$, de acuerdo al $\mathrm{CIAT}^{[7]}$.

- La identificación de cada etapa se hizo en base a un código que consta de una letra y un número. La letra corresponde a la inicial de la fase a la cual pertenece la etapa particular. Es decir, $V$ si pertenece a la fase vegetativa, o R si pertenece a la fase reproductiva. El número indica la posición de la etapa en la escala (Figura No. 1).

Figura No. 1. Etapa de desarrollo del cultivo de frijol. Fuente: INTA (2004)

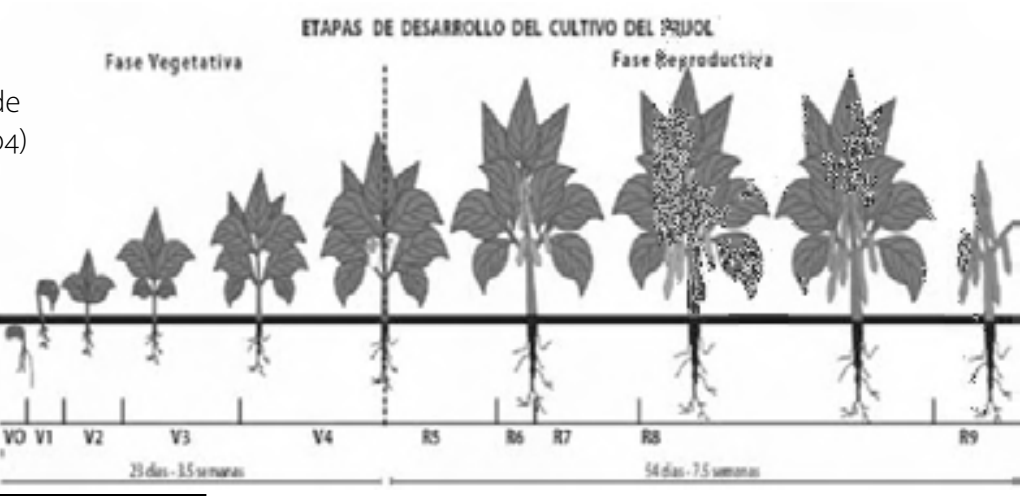

${ }^{[6]}$ Días después de la siembra.

${ }^{[7]}$ Centro Internacional de Agricultura Tropical. 


\subsection{Diseño experimental}

El ensayo fue un diseño bifactorial en bloques completos al azar (DBCA), con 4 tratamientos y 3 repeticiones. Se estudiaron 2 factores; el factor A y el factor B, y fue analizado bajo un modelo lineal de $\mathrm{DBCA}^{[8]}$, en inicio porque, el diseño no presentó las unidades experimentales adecuadas y en segundo, porque se pretendió analizar un factor por separado y, no el cambio de un factor según los niveles de los otros factores o su interacción. A continuación se sintetiza.

FACTOR A: variedad de frijol<smiles>[CH]</smiles>

Niveles $A_{1}$ : DOR-364 variedad de frijol mejorado $A_{2}$ : H-46 variedad de frijol mejorado
FACTOR B: método de siembra

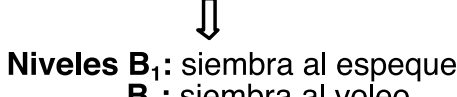

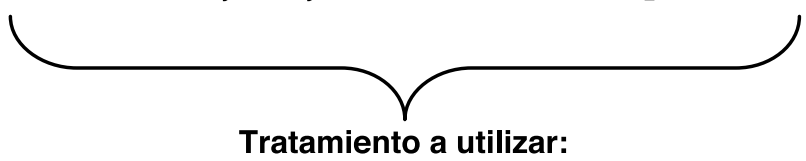

A1B1 (A): variedad DOR-364 - siembra al espeque

A1B2 (B): variedad DOR-364 - siembra al voleo

A2B1 (C): variedad $\mathrm{H}-46$ - siembra al espeque

A2B2 (D): Variedad H-46 - siembra al voleo

\section{Variables a evaluar}

1) En la etapa vegetativa:

a) Germinación: A la par de la parcela experimental se estableció una parcela de $2 \mathrm{~m}^{2}$, la cual contenía dos surcos cada uno de ellos con 100 semillas. Estas se evaluaron tomando en cuenta el número de plántulas de cada variedad a los 10 (DDS).

b) Altura (cm) de las plantas: Se midió con una cinta milimetrada, desde la base del tallo hasta la inserción de las hojas más tiernas.

c) Diámetro ( $\mathrm{mm})$ del tallo: Se midió con verniere, desde los tres centímetros del nivel el suelo hasta la altura de la huella de los cotiledones.

d) Número de hojas por plantas: Se verificó de manera visual, mediante el conteo de cada planta evaluada.

2) En la tapa reproductiva:

a) Número de flores por plantas: Se efectuó mediante el conteo de cada planta evaluada de forma visual (una vez en estado de prefloración, $\mathrm{R}_{5}$ ) hasta la formación de vainas, $\mathrm{R} 7$ ), para determinar el número de futuras vainas formadas.

\footnotetext{
${ }^{[8]}$ Diseño de Bloques Completamente Al Azar
} 
b) Número de vainas por planta: Se realizó mediante el conteo de forma visual desde la formación de las vainas hasta la maduración. cuando se va a enumerar: frijol, maíz, etc.

c) Longitud de vainas $(\mathrm{cm})$ por plantas: Se realizó una sola medición en el momento de la cosecha, desde el ápice hasta el pedicelo, la cual se utilizó una cinta milimetrada.

d) Número de granos por vainas por plantas: Se efectuó en el momento de la cosecha a través del conteo de granos por vainas de forma manual, obteniéndose el promedio de granos por vainas por tratamientos.

e) Peso de la semilla: Una vez desgranada las vainas se tomaron en cuenta únicamente las vainas de la parcela útil (P.U), las cuales fueron pesadas en una balanza electrónica; determinando de esta forma el rendimiento $(\mathrm{g} / \mathrm{P} . \mathrm{U})^{[9]}$.

\section{Resultados y discusión}

\subsection{Caracterización del método de siembra tradicional (al voleo y al espeque)}

Las labores de siembra para los dos métodos se iniciaron con:

- Eliminación parcial de la vegetación o matorral y limpieza de tocones (troncos) de los árboles tumbados por el huracán Félix.

- Se hizo la desinfección natural por 4 días soleados de la maleza cortada.

- Posteriormente se procedió lanzar sobre la maleza 5 manojos de semilla en cada parcela, la cual fue cortada, picada y tapada con función de una cama se siembra, en el caso del método al voleo (Foto 2).

- En cuanto al método al espeque, se efectuó la siembra directa que consistió en roturar el suelo con una vara de madera con punta, para luego depositar de 3 - 4 semillas por golpe o hoyo, con espaciamiento de $15 \mathrm{~cm}$ entre golpes de siembra y $40-60 \mathrm{~cm}$ entre surcos (Foto 3 ).
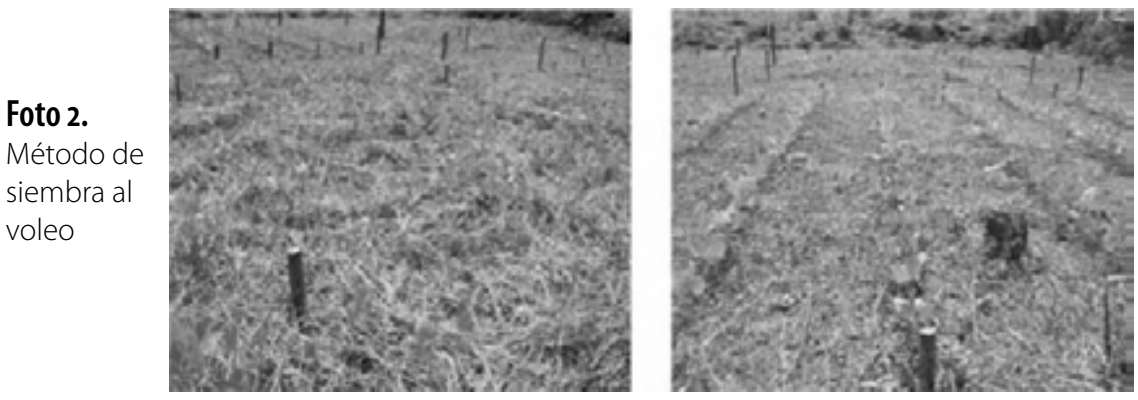

Foto 3. Método de siembra al espeque

${ }^{[9]}$ Grano por Parcela Útil 


\subsection{Análisis de las características morfométricas en las diferentes etapas del cultivo (vegetativa y reproductiva)}

\subsubsection{Etapa vegetativa}

\subsubsection{Germinación y descripción morfométrica de la variable altura}

La prueba germinativa para las dos variedades en estudio, presentaron una alta viabilidad en la semilla, que fueron estimadas a los 10 DDS (Gráfico No. 1). En cuanto al (Gráfico No. 2) se aprecia que la altura bajo tratamiento $\mathrm{D}\left(\mathrm{A}_{2} \mathrm{~B}_{2}\right)$ para los períodos evaluados (13, 20 y $27 \mathrm{DDS}$ ) presentan las mejores medias; seguido del tratamiento $\mathrm{B}\left(\mathrm{A}_{1} \mathrm{~B}_{2}\right)$.

Estos valores gráficados manifiestan medias altas por cada tratamiento; las cuales, pueden deberse a las labores agrícolas establecidas, como por ejemplo:

- La preparación del suelo (desinfección natural).

- Densidad de siembra.

- Suministro contínuo de agua.

- Control permanente de maleza, entre otras labores.

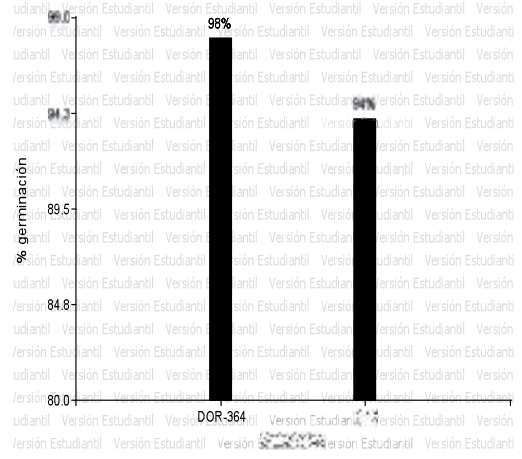

Gráfico No. 1. Porcentaje germinativo de las dos variedades

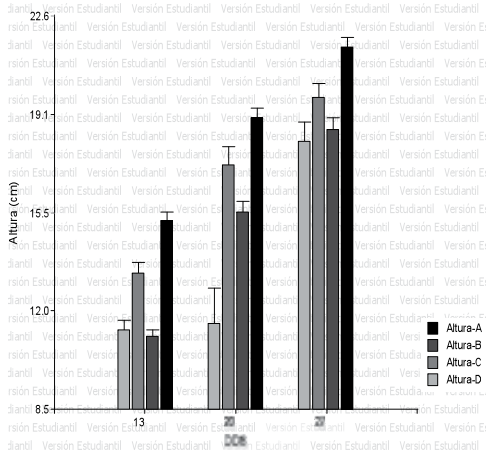

Gráfico No. 2. comportamiento de la variable altura $(\mathrm{cm})$ para los períodos 13,20 y 27 DDS, según tratamientos.

\subsubsection{Descripción morfométrica de la variable diámetro y hojas/planta}

En el gráfico No. 3, muestra similitudes diamétricas en los cuatro tratamientos para los períodos 13 y 27 DDS, a excepción del período 20 DDS, donde despunta la media del tratamiento $\mathrm{A}\left(\mathrm{A}_{1} \mathrm{~B}_{1}\right)$. Los resultados ponen de manifiesto, que la variedad diamétrica tuvo efecto entre los tratamientos, lo que posiblemente se deban a las labores agrícolas establecidas, destacándose el control continuo de malezas y el suministro de agua.

En cuanto al gráfico No. 4, de manera generalizada se muestra un comportamiento similar en cada período, con una leve diferencia de medias en el período 20 DDS para el tratamiento $\mathrm{A}\left(\mathrm{A}_{1} \mathrm{~B}_{1}\right)$. De acuerdo a las observaciones de campo, esta leve 
diferencia, probablemente se deba: a las mayores caídas de las hojas por efecto del viento y al marchitamiento provocado por la luz solar en los tratamientos $B\left(\mathrm{~A}_{1} \mathrm{~B}_{2}\right)$, $\mathrm{C}\left(\mathrm{A}_{2} \mathrm{~B}_{1}\right)$ y $\mathrm{D}\left(\mathrm{A}_{2} \mathrm{~B}_{2}\right)$.

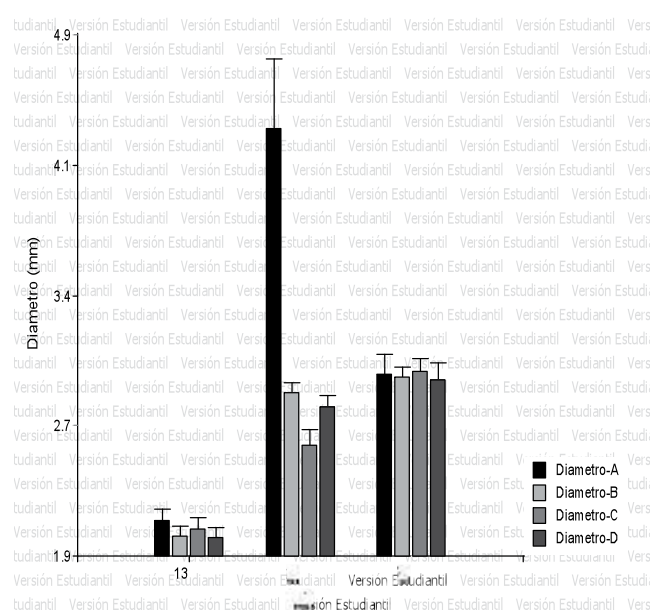

Gráfico No. 3. Comportamiento de la variable diámetro $(\mathrm{mm})$ para los períodos 13, 20 y 27 DDS, según tratamientos.

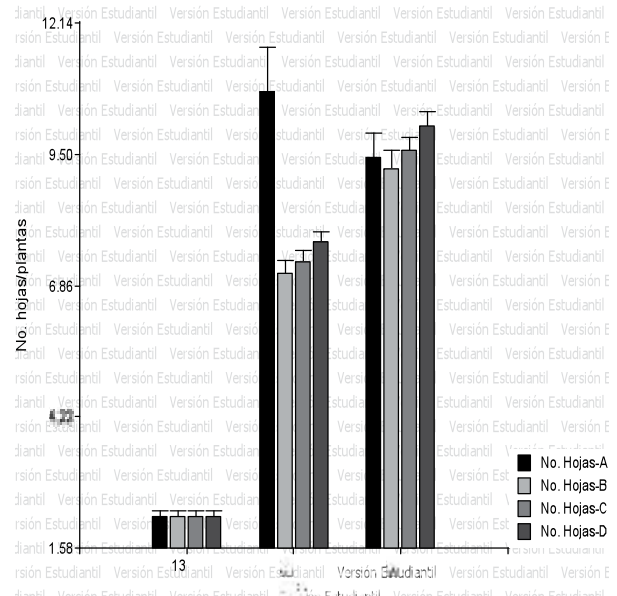

Gráfico No. 4. Comportamiento de la variable No. hojas/plantas para los períodos 13, 20 y 27 DDS, según tratamientos.

\subsubsection{Prueba estadística de la variable altura, diámetro}

La prueba mostró diferencia estadística en las variables: altura y diámetro versus los tratamientos $(\mathrm{p}=<0.0001)$ y $(\mathrm{p}=0.0187)$; despuntando el tratamiento $\mathrm{D}$, con las mayores medias (240.21) de Altura (gráfica No. 5) y (207.66) de diámetro (gráfica No. 6).

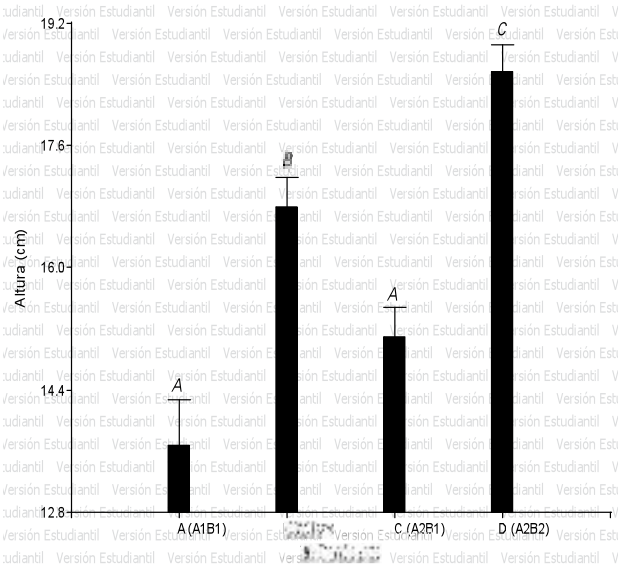

Gráfico No. 5. Altura (cm) vs tratamientos

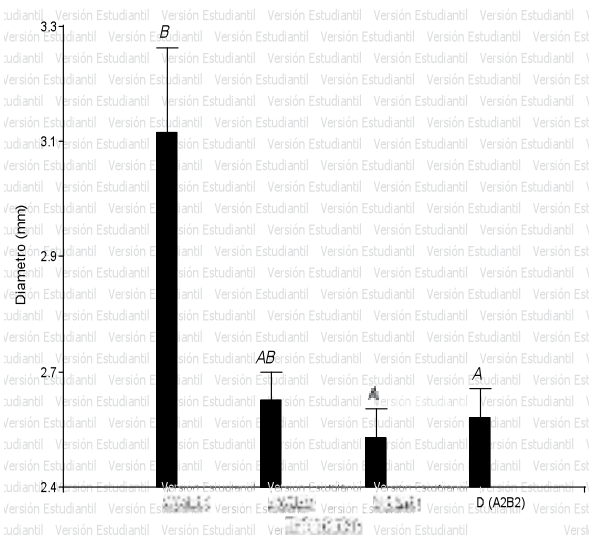

Gráfico No. 6. Diámetro ( $\mathrm{mm}$ ) vs tratamientos 


\subsubsection{Prueba estadística de la variable No. de hojas/planta}

En caso de la variable No. De hojas/planta, la prueba no reveló diferencias estadísticas significativas entre medias versus tratamientos $(\mathrm{p}=0.4547)$; lo cual indican que la caída de las hojas y al marchitamiento presentado se debió a un proceso normal del cultivo.

\subsubsection{Etapa reproductiva}

\subsubsection{Descripción morfométrica de la variable No. flores/plantas y No. de vainas por planta}

El gráfico No. 7, muestra que el período de máximo desarrollo floral fue a los 48 DDS. Posterior a este período floral se observa una disminución en la formación de flores por plantas (55 DDS).

En este período se destacó el manejo agronómico anticipado o preventivo como fue:

- La utilización de semilla mejorada libre de patógenos.

- El uso de una variedad resistente a plagas y enfermedades.

- El control continuo de malezas.

En cuanto a la formación de vainas inicio a los 48 DDS, despuntando el tratamiento $C\left(\mathrm{~A}_{2} \mathrm{~B}_{1}\right)$, en relación a los demás tratamientos. Del mismo modo, a los 55 y 70 DDS se observa entre los tratamientos $\mathrm{A}\left(\mathrm{A}_{1} \mathrm{~B}_{1}\right), \mathrm{C}\left(\mathrm{A}_{2} \mathrm{~B}_{1}\right)$ y $\mathrm{D}\left(\mathrm{A}_{2} \mathrm{~B}_{2}\right)$ una semejanza en la cantidad de vainas formadas por plantas, no así, en el tratamiento $B\left(\mathrm{~A}_{1} \mathrm{~B}_{2}\right)$ (gráfico No. 8).

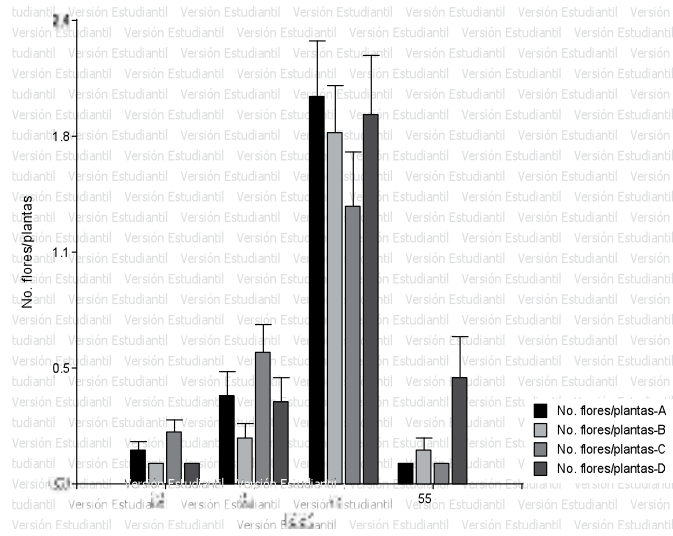

Gráfico No. 7. Comportamiento de la variable No. flores/plantas para los periodos 34, 41, 48 y 55 DDS, según tratamientos.

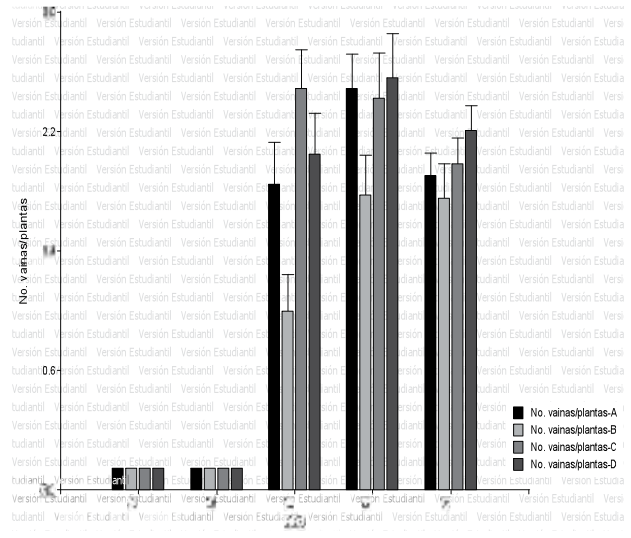

Gráfico No. 8. Comportamiento de la variable No. vainas/plantas para los periodos 34, 41, 48, 55 y 70 DDS, según tratamientos. 


\subsubsection{Descripción morfométrica de la variable long. vainas/plantas y No. de granos/vaina/planta}

El gráfico No. 9, modelo igualdad de comportamiento en los tratamientos $\mathrm{A}\left(\mathrm{A}_{1} \mathrm{~B}_{1}\right)$ y $C\left(\mathrm{~A}_{2} \mathrm{~B}_{1}\right)$, con un despunte del tratamiento $\mathrm{B}\left(\mathrm{A}_{1} \mathrm{~B}_{2}\right)$, en relación al tratamiento $\mathrm{D}$ $\left(\mathrm{A}_{2} \mathrm{~B}_{2}\right)$. Para este período las labores agrícolas que prevalecieron fueron: el control de malezas de forma manual y el suministro de agua.

En cuanto al gráfico No. 10, la distribución de medias de la variable No. granos/ vainas/plantas versus tratamientos, exhibe que los tratamientos $\mathrm{A}\left(\mathrm{A}_{1} \mathrm{~B}_{1}\right)$ y $\mathrm{D}\left(\mathrm{A}_{2} \mathrm{~B}_{2}\right)$ presentan las mejores medias en relación a los tratamientos $B\left(\mathrm{~A}_{1} \mathrm{~B}_{2}\right)$ y $C\left(\mathrm{~A}_{2} \mathrm{~B}_{1}\right)$.

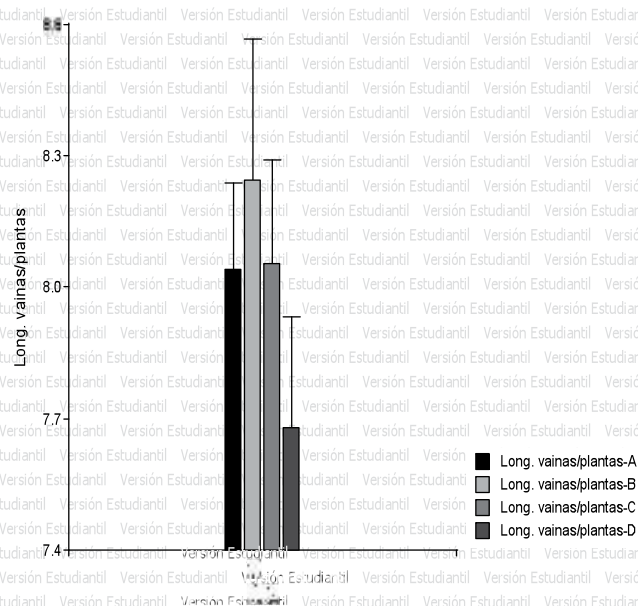

Gráfico No. 9. Comportamiento de la variable long. flores/plantas (cm) para el periodo 70 DDS, según tratamientos.

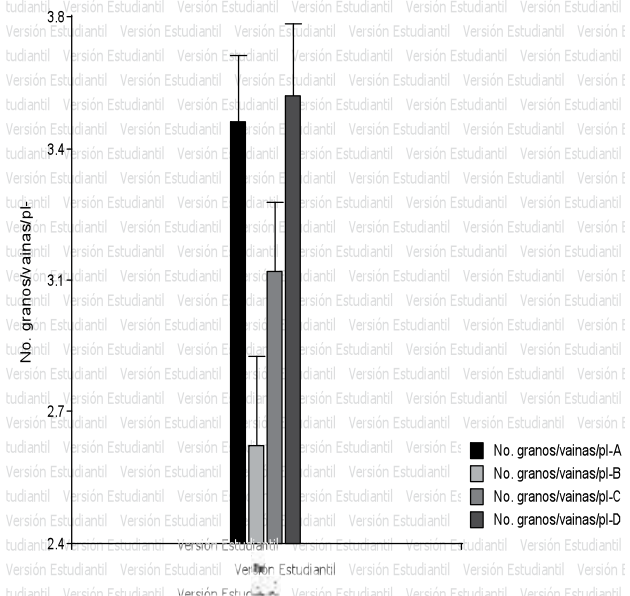

Gráfico No. 10. Comportamiento de la variable No. vainas/planta para el periodo 70 DDS, según tratamientos.

\subsubsection{Prueba estadística de la variable No. de flores/planta y long. Vaina/planta}

La prueba estadística no encontró diferencias en la variable No. de flores/planta y long. Vainas/plantas, aunque en el gráfico No. 9 se observa una desigualdad considerable de medias entre los tratamientos $\mathrm{B}\left(\mathrm{A}_{1} \mathrm{~B}_{2}\right)$ y $\mathrm{D}\left(\mathrm{A}_{2} \mathrm{~B}_{2}\right)$.

\subsubsection{Prueba estadística de la variable No. de vaina/planta y No. Granos/vaina/planta}

La prueba muestra diferencias estadísticas en ambas variables entre el tratamiento, prevaleciendo los dos últimos tratamientos (C y D), en el caso de la variable No. Vaina por planta (gráfica No. 11)

En cuanto a la variable No. De granos/vaina/planta (gráfica No. 12) los tratamientos $\mathrm{A}\left(\mathrm{A}_{1} \mathrm{~B}_{1}\right)$ y $\mathrm{D}\left(\mathrm{A}_{2} \mathrm{~B}_{2}\right)$, presentan las mayores cantidades de granos por vainas en las 
plantas, con promedios de 70 y 70.62 ; seguido el tratamiento $C\left(\mathrm{~A}_{2} \mathrm{~B}_{1}\right)$ con promedio de 56.23 (gráfica No. 12). En base a los promedios de la variable No. granos/vainas/ plantas, podemos pronosticar que los mayores rendimientos se prevén obtener en los tratamientos $\mathrm{A}\left(\mathrm{A}_{1} \mathrm{~B}_{1}\right)$ y $\mathrm{D}\left(\mathrm{A}_{2} \mathrm{~B}_{2}\right)$, siempre y cuando las actividades de post cosecha se efectúen sin el menor riesgo posible, tales como: pre secado, aporreo, secado y almacenamiento.

\subsection{Evaluación del rendimiento de las variables mejoradas DOR-364 y H-46, bajo diseño experimental DBCA}

\subsubsection{Implicancia de la evaluación de la variable rendimiento a través del análisis ANAVA, en el DBCA}

En base a la prueba de hipótesis de bloques, de tratamientos e hipótesis de contrastes ortogonales entre tratamientos, así como, los resultados obtenidos en el análisis de la varianza, podemos afirmar con un nivel de confiabilidad del 95 por ciento, que las medias de las variables entre tratamientos difieren estadísticamente entre sí, y que

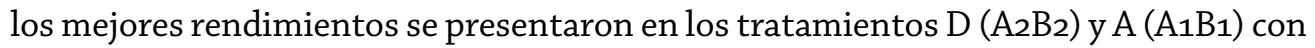
promedios de 77.63 y 71.33; por lo tanto se recomienda la variedad $\mathrm{H}-46$ sembrada al voleo y la variedad DOR-364 sembrada al espeque. A continuación se ilustra los rendimientos obtenidos por bloques y tratamientos; las cuales son comprobados con los rendimientos máximos y mínimos en el Pacífico de Nicaragua y la RAAN (gráfico No. 13)

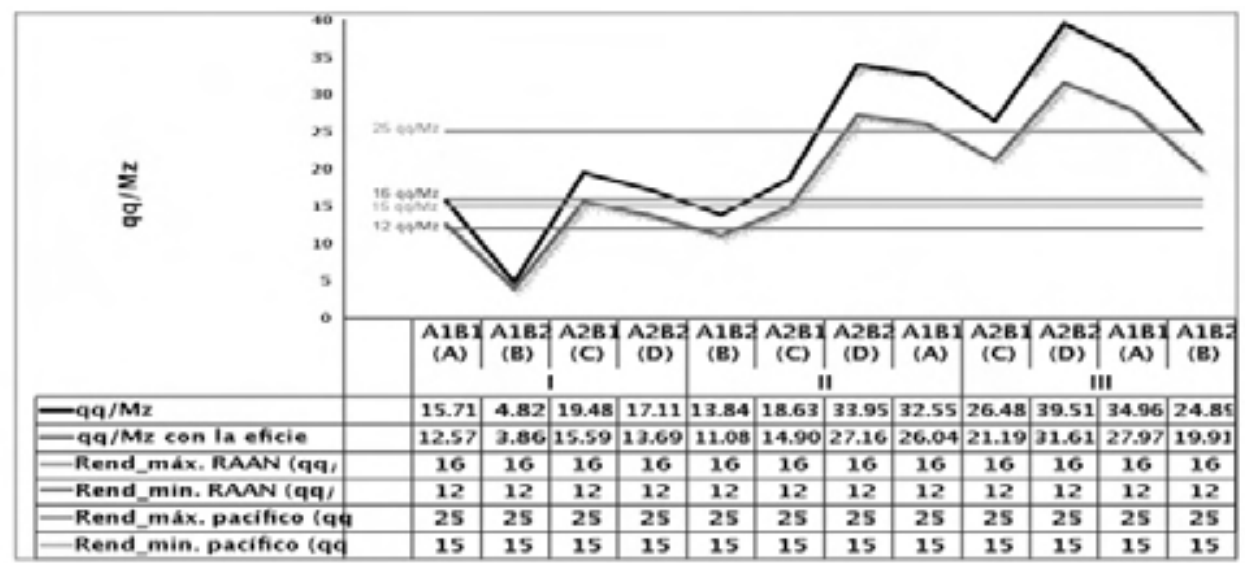

Gráfico No. 13. Rendimiento obtenido por cada tratamiento en cada bloque. Los rendimientos obtenidos fueron estimados según metodología del INTA publicada para el año 2004.

De acuerdo a la gráfica No. 13, los rendimientos estimados por el INTA y en base a los cálculos matemáticos realizados por bloques y tratamientos, manifiestan que los rendimientos del tratamiento $C$ del bloque I estima rendimientos máximos de la RAAN con $15.59 \mathrm{qq} / \mathrm{mz}$, en caso del bloque II el tratamiento D estima rendimientos máximos del Pacífico con $27.16 \mathrm{qq} / \mathrm{mz}$, al igual en el bloque III con 31.61 qq/mz. 
A continuación se muestran los rendimientos obtenidos por tratamiento, agrupados por bloques:

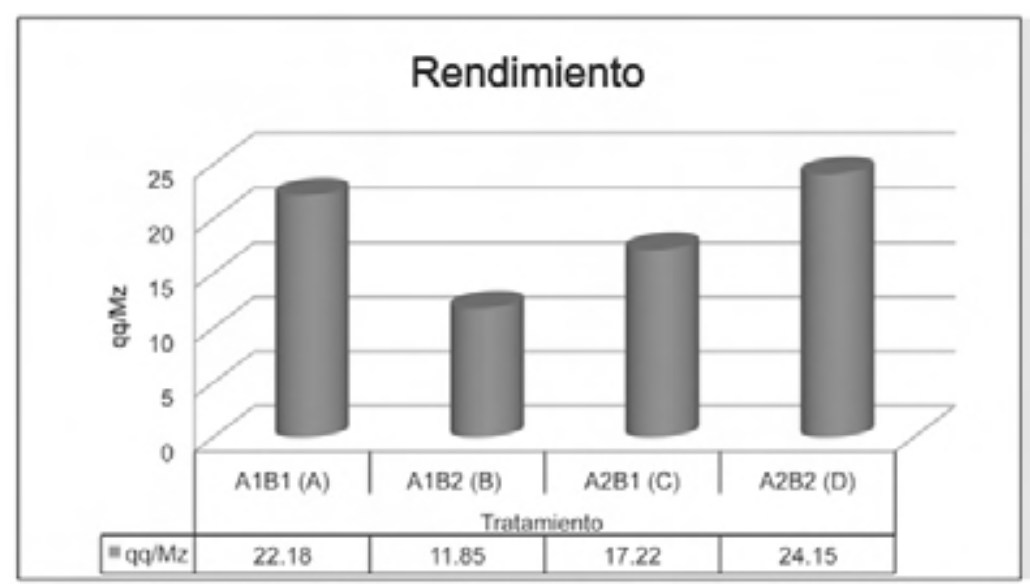

Gráfico No. 14. Rendimiento obtenido qq/Mz por tratamiento

De acuerdo a la gráfica No. 14, los rendimientos obtenidos prevalecen en los tratamientos $\mathrm{A}\left(\mathrm{A}_{1} \mathrm{~B}_{1}\right)$ y $\mathrm{D}\left(\mathrm{A}_{2} \mathrm{~B}_{2}\right)$, con los mayores rendimientos con 22.18 y $24.15 \mathrm{qq} / \mathrm{Mz}$ que están estimadas por los rendimientos máximos del Pacífico y mayores de la RAAN.

\section{Conclusiones}

En la etapa vegetativa emergencia (13 DDS) y crecimiento (20 y 27 DDS), se encontraron diferencias estadísticas entre tratamientos en las variables altura $(\mathrm{cm})$ y diámetro $(\mathrm{cm})$, no así en la variable número de hojas/plantas. En la variable altura sobresalió la variedad H-46 sembrado al voleo con promedio de 240.20 y en la variable diamétrica $(\mathrm{mm})$ la variedad DOR-364 sembrado al espeque y al voleo con 207.66 y 178.08 de promedio.

En la etapa reproductiva las variables número de vainas/plantas y granos/vainas/ plantas presentaron diferencias estadísticas entre tratamiento, contrario a las variables No. flores/plantas y long. vainas/plantas que no mostraron diferencias. En la variable No. vainas/plantas sobresalen las variedades DOR-364 sembrado al espeque y la variedad H-46 sembrado al espeque y al voleo con promedios de 296.07, $304.97 \mathrm{y}$ 307.53, y en la variable No. granos/vainas/plantas las variedades DOR-364 sembrado al espeque y la variedad H-46 sembrado al voleo con promedios de 70 y 70.62 .

El análisis de ANAVA, con una confiabilidad del 95 por ciento concluye que las medias de la variable rendimiento entre tratamientos difieren estadísticamente, por lo que se recomiendan, la variedad H-46 sembrada al voleo y la variedad DOR-364 sembrada al espeque, ya que presentaron los mayores rendimientos. 


\section{Lista de referencia}

Centro Internacional de Agricultura Tropical (1984). Etapa de desarrollo de la planta de frijol común. Disponible en: http://www.books.google.com.ni/books?id=A2SJrDrokqIC...

Fernández, E.; Brooks, M. (2008). Estudio biofísico de la finca "Snaki" de la universidad URACCAN, comunidad de Moss, municipio de Waspam río Coco, RAAN. Monografía Ing. URACCAN. 20-40 p.

INETER (2010). Departamento estadística de meteorología. Disponible en: http://www. ineter.gob.ni

InfoStat. (2010). Manual de usuario. Grupo InfoStat, FCA, Universidad Nacional de Córdoba. 1 ed. Editorial Brujas Argentina. 318 p.

INTA (1996). Guía Técnica de Granos Básicos.

Mairena, Z.; Potecto, J. (2004). Evaluación del comportamiento de cuatro variedades de frijol (Phaseolus vulgaris L.), bajo cuatro dosis de fertilizante fosfórico en la comunidad indígena de Bum Sirpi, RAAN, Nicaragua. Tesis Ing. BICU-CIUM. 83 p.

Mendiburu, F. (2007). Diseños experimentales. Disponible en: http://tarwi.lamolina.edu. pe/ fmendiburu

Ministerio de Agricultura y Ganadería (1991). Aspectos técnicos sobre cuarenta y cinco cultivos agrícolas de Costa RICA. Dirección general de investigación y extensión agrícola, San José, Costa Rica. Disponible en: http://www.mag.go.cr/bibioteca_virtual_ciencia/tec_frijol.pdf

Padilla, M.; Antonio, D. (2003). Percepción de los pueblos Miskitus de Waspam río Coco sobre la introducción de seis variedades mejoradas de frijol (Phaseolus vulgaris). Monografía Ing. RAAN, Nicaragua, URACCAN. 39 p.

Sampieri, H; Collado, F; Lucio, B, (1997). Metodología de la investigación. Cuarta edición. MacGraw Hill, Interamericana. México. 128 p.

Tapia Barquero, H; Camacho Henriquez. A. (1988). Manejo Integrado de la Producción de Frijol basado en labranza cero. Managua.Nicaragua.181p.

White, J. W. (1985). Conceptos básicos de fisiología del frijol. Ing. López, M.I. Fernández., Avan Schoonhoven (ed). Frijol: Investigación y Producción. CIAT. Cali. Colombia, p. 43.60 . 